\title{
A New Iterative Scheme of Modified Mann Iteration in Banach Space
}

\author{
Jinzuo Chen, Dingping Wu, and Caifen Zhang \\ College of Applied Mathematics, Chengdu University of Information Technology, No. 24, Block 1, Xuefu Road, Chengdu 610225, China \\ Correspondence should be addressed to Jinzuo Chen; chanjanegeoger@163.com
}

Received 5 October 2013; Revised 28 December 2013; Accepted 29 December 2013; Published 11 February 2014

Academic Editor: Sehie Park

Copyright (c) 2014 Jinzuo Chen et al. This is an open access article distributed under the Creative Commons Attribution License, which permits unrestricted use, distribution, and reproduction in any medium, provided the original work is properly cited.

\begin{abstract}
We introduce the modified iterations of Mann's type for nonexpansive mappings and asymptotically nonexpansive mappings to have the strong convergence in a uniformly convex Banach space. We study approximation of common fixed point of asymptotically nonexpansive mappings in Banach space by using a new iterative scheme. Applications to the accretive operators are also included.
\end{abstract}

\section{Introduction}

Let $E$ be a real Banach space, $C$ a nonempty closed convex subset of $E$, and $T: C \rightarrow C$ a mapping. Recall that $T$ is a nonexpansive mapping [1] if $\|T x-T y\| \leq\|x-y\|$ for all $x, y \in C$, and $T$ is asymptotically nonexpansive [2] if there exists a sequence $\left\{k_{n}\right\}$ with $k_{n} \geq 1$ for all $n$ and $\lim _{n \rightarrow \infty} k_{n}=1$ such that $\left\|T^{n} x-T^{n} y\right\| \leq k_{n}\|x-y\|$ for all integers $n \geq 1$ and $x, y \in C$. A point $x \in C$ is a fixed point of $T$ provided $T x=x$. Denote by $\operatorname{Fix}(T)$ the set of fixed points of $T$; that is, $\operatorname{Fix}(T)=\{x \in C: T x=x\}$.

Iterative methods are often used to solve the fixed point equation $T x=x$. One classical iteration process is introduced in 1953 by Mann [3] which is well known as Mann iteration process and is defined as follows:

$$
x_{n+1}=\alpha_{n} x_{n}+\left(1-\alpha_{n}\right) T x_{n}, \quad n \geq 0,
$$

where the sequence $\left\{\alpha_{n}\right\}$ is chosen in $(0,1)$ and the initial guess $x_{0} \in C$ is arbitrarily chosen.

There exists rich literature on the convergence of Mann iteration for different classes of operators considered on various spaces. Mann's iteration method (1) has been proved to be a powerful method for solving nonlinear operator equations involving nonexpansive mapping, asymptotically nonexpansive mapping, and other kinds of nonlinear mapping; see [3-11] and the references therein.
It is known that Mann's iteration method (1) is in general not strongly convergent for nonexpansive mappings. So to get strong convergence, one has to modify the iteration method (1). In this regard, we will show the modified iteration in Section 3.

Motivated and inspired by the research going on in these fields, we suggest and analyze now new modified Mann's iteration for finding the common fixed point of the nonexpansive mappings and asymptotically nonexpansive mappings in Banach space. We propose the modified Mann's iteration and consider the strong convergence of the approximate solutions for nonexpansive and asymptotically nonexpansive in Banach space.

We suggest and analyze the following iterative:

$$
\begin{aligned}
& x_{0} \in C \quad \text { chosen arbitrarily, } \\
& y_{n}=\beta_{n} x_{n}+\left(1-\beta_{n}\right) T x_{n}, \\
& z_{n}=\gamma_{n} x_{n}+\left(1-\gamma_{n}\right) S x_{n}, \\
& x_{n+1}=\alpha_{n} y_{n}+\left(1-\alpha_{n}\right) R z_{n}, \quad n \geq 0, \\
& x_{0} \in C \quad \text { chosen arbitrarily, } \\
& y_{n}=\beta_{n} x_{n}+\left(1-\beta_{n}\right) T^{n} x_{n}, \\
& z_{n}=\gamma_{n} x_{n}+\left(1-\gamma_{n}\right) S^{n} x_{n}, \\
& x_{n+1}=\alpha_{n} y_{n}+\left(1-\alpha_{n}\right) z_{n}, \quad n \geq 0,
\end{aligned}
$$


$x_{0} \in C$ chosen arbitrarily,

$$
\begin{aligned}
& y_{n}=\beta_{n} x_{n}+\left(1-\beta_{n}\right) J_{1, n} x_{n}, \\
& z_{n}=\gamma_{n} x_{n}+\left(1-\gamma_{n}\right) J_{2, n} x_{n}, \\
& x_{n+1}=\alpha_{n} y_{n}+\left(1-\alpha_{n}\right) J_{3, n} z_{n}, \quad n \geq 0,
\end{aligned}
$$

and if there exists two sequences $\left\{x_{n}^{\prime}\right\}$ and $\left\{x_{n}^{\prime \prime}\right\}$ generated by

$x_{0} \in C$ chosen arbitrarily,

$$
\begin{aligned}
& y_{n}=\beta_{n} x_{n}^{\prime}+\left(1-\beta_{n}\right) T^{n} x_{n}^{\prime}, \\
& z_{n}=\gamma_{n} x_{n}^{\prime \prime}+\left(1-\gamma_{n}\right) S^{n} x_{n}^{\prime \prime}, \\
& x_{n+1}^{\prime}=\alpha_{n} z_{n}+\left(1-\alpha_{n}\right) y_{n}, \\
& x_{n+1}^{\prime \prime}=\alpha_{n} y_{n}+\left(1-\alpha_{n}\right) z_{n}, \quad n \geq 0, \\
& x_{0} \in C \quad \text { chosen arbitrarily, } \\
& y_{n}=\beta_{n} x_{n}^{\prime}+\left(1-\beta_{n}\right) J_{1, n} x_{n}^{\prime}, \\
& z_{n}=\gamma_{n} x_{n}^{\prime \prime}+\left(1-\gamma_{n}\right) J_{2, n} x_{n}^{\prime \prime}, \\
& x_{n+1}^{\prime}=\alpha_{n} z_{n}+\left(1-\alpha_{n}\right) y_{n}, \\
& x_{n+1}^{\prime \prime}=\alpha_{n} y_{n}+\left(1-\alpha_{n}\right) z_{n}, n \geq 0 .
\end{aligned}
$$

Our second modification of Mann's iteration method (1) is adaption to (2) for finding a zero of an $m$-accretive operator $A$, for which we assume that the zero set $A^{-1}(0) \neq \emptyset$. Our iterations process $\left\{x_{n}\right\}$ is given by (4), and sequences $\left\{x_{n}^{\prime}\right\}$ and $\left\{x_{n}^{\prime \prime}\right\}$ are as follows (6), where, for each $r>0$, $J_{r}=(I+r A)^{-1}$ is the resolvent of $A$. We prove that not only $\left\{x_{n}\right\}_{n}^{\infty}$ defined by (4) but also $\left\{x_{n}^{\prime}\right\}$ and $\left\{x_{n}^{\prime \prime}\right\}$ generated by (6) converge strongly to a zero of $A$ under certain assumptions in a uniformly Banach space.

We write $x_{n} \rightarrow x$ to indicate that the sequence $\left\{x_{n}\right\}$ converges strongly to $x$. Using $F$ is to denote the set of common fixed point of the mappings $T, S$, and $R$, and using $\bar{F}$ is to denote the set of common fixed points of the mappings $T$ and $S$.

\section{Preliminaries}

This section collects some lemmas, which will be used in the proofs for the main results in the next section.

Lemma 1 (see [8]). Let $\left\{a_{n}\right\},\left\{b_{n}\right\}$, and $\left\{\delta_{n}\right\}$ be sequences of nonnegative real numbers satisfying the inequality

$$
a_{n+1} \leq\left(1+\delta_{n}\right) a_{n}+b_{n}, \quad n \geq 1 .
$$

If $\sum_{n=1}^{\infty} \delta_{n}<\infty$ and $\sum_{n=1}^{\infty} b_{n}<\infty$, then

(1) $\lim _{n \rightarrow \infty} a_{n}$ exists;

(2) $\lim _{n \rightarrow \infty} a_{n}=0$ whenever $\liminf _{n \rightarrow \infty} a_{n}=0$.

Lemma 2 (see [12]). Suppose that $E$ is a uniformly convex Banach space and $0<t_{n}<1$ for all $n \in N$. Let $\left\{x_{n}\right\}$ and $\left\{y_{n}\right\}$ be two sequences of E such that $\limsup _{n \rightarrow \infty}\left\|x_{n}\right\| \leq r$, $\limsup _{n \rightarrow \infty}\left\|y_{n}\right\| \leq r$ and $\lim _{n \rightarrow \infty}\left\|t_{n} x_{n}+\left(1-t_{n}\right) y_{n}\right\|=r$ hold for some $r \geq 0$; then $\lim _{n \rightarrow \infty}\left\|x_{n}-y_{n}\right\|=0$.

Lemma 3 (see [10]). A mapping $T: C \rightarrow C$ with a nonempty fixed point set $F$ in $C$ will be said to satisfy Condition (I).

If there is a nondecreasing function $f:[0, \infty) \rightarrow[0, \infty)$ with $f(0)=0, f(r)>0$ for all $r \in(0, \infty)$ such that $\|x-T x\| \geq$ $f(d(x, F))$ for all $x \in C$, where $d(x, F)=\inf \{\|x-p\|: p \in F\}$.

Lemma 4 (see [13]). Given a number $r>0$, let $E$ be a uniformly convex Banach space; then there exists a continuous strictly increasing function $\varphi:[0, \infty] \rightarrow[0, \infty]$ with $\varphi(0)=$ 0 , such that

$$
\begin{aligned}
\|\lambda x+\mu y+\gamma z\|^{2} \leq & \lambda\|x\|^{2}+\mu\|y\|^{2} \\
& +\gamma\|z\|^{2}-\lambda \mu \varphi(\|x-y\|),
\end{aligned}
$$

for all $\lambda, \mu, \gamma \in[0,1]$ and $x, y, z \in$ Esuch that $\|x\| \leq r,\|y\| \leq r$ and $\|z\| \leq r$.

Lemma 5 (see [14]). Let $\left\{\alpha_{n}\right\},\left\{\beta_{n}\right\}$ be sequences of nonnegative real numbers such that $\sum_{n=1}^{\infty} \alpha_{n}=\infty$. If $\sum_{n=1}^{\infty} \alpha_{n} \beta_{n}<\infty$, then $\liminf _{n \rightarrow \infty} \beta_{n}=0$.

Lemma 6 (see [15]). For $\lambda>0, \mu>0$, and $x \in E$, the following identity holds

$$
J_{\lambda} x=J_{\mu}\left(\frac{\mu}{\lambda}+\left(1-\frac{\mu}{\lambda}\right) J_{\lambda} x\right) .
$$

\section{Convergence to a Common Fixed Point of Nonexpansive Mappings}

In this part, we prove our main theorem for finding a common fixed point of nonexpansive mappings in Banach space.

Theorem 7. Let $C$ be a nonempty closed convex subset of a uniformly convex Banach space $E$, and let $T, S$, and $R$ be three nonexpansive commuting mappings of $C$ satisfying Condition (I) and $F \neq \emptyset$. Given that $\left\{\alpha_{n}\right\},\left\{\beta_{n}\right\}$, and $\left\{\gamma_{n}\right\}$ are sequences in $(0,1)$ such that $\sum \alpha_{n}<\infty, \sum \gamma_{n} \beta_{n}=\infty, \sum\left(1-\gamma_{n}\right)<\infty$ for all $n \geq 1$.

Define a sequence $\left\{x_{n}\right\}_{n=0}^{\infty}$ in $C$ by algorithm (2); then $\left\{x_{n}\right\}_{n=0}^{\infty}$ strongly converges to a common fixed point of T, $S$, and $R$.

Proof. First, we observe that $\left\{x_{n}\right\}$ is bounded; if we take an arbitrary fixed point $q$ of $F$, noting that $\left\|y_{n}-q\right\| \leq\left\|x_{n}-q\right\|$ and $\left\|z_{n}-q\right\| \leq\left\|x_{n}-q\right\|$, we have

$$
\begin{aligned}
\left\|x_{n+1}-q\right\| & =\left\|\alpha_{n} y_{n}+\left(1-\alpha_{n}\right) R z_{n}-q\right\| \\
& \leq \alpha_{n}\left\|y_{n}-R z_{n}\right\|+\left\|R z_{n}-q\right\| \\
& \leq\left(1+2 \alpha_{n}\right)\left\|x_{n}-q\right\| .
\end{aligned}
$$

By Lemma 1 and $\sum \alpha_{n}<\infty, \lim _{n \rightarrow \infty}\left\|x_{n}-q\right\|$ exists. Denote

$$
\lim _{n \rightarrow \infty}\left\|x_{n}-q\right\|=c \text {. }
$$


Hence, $\left\{x_{n}\right\}$ is bounded, so are $\left\{y_{n}\right\}$ and $\left\{z_{n}\right\}$. Now

$$
\begin{aligned}
\left\|x_{n+1}-q\right\| & =\left\|\alpha_{n} y_{n}+\left(1-\alpha_{n}\right) R z_{n}-q\right\| \\
& \leq \alpha_{n}\left\|y_{n}-R z_{n}\right\|+\left\|z_{n}-q\right\| .
\end{aligned}
$$

Since $\left\|z_{n}-q\right\| \leq\left\|x_{n}-q\right\|$, this implies that

$$
\lim _{n \rightarrow \infty}\left\|z_{n}-q\right\|=\lim _{n \rightarrow \infty}\left\|x_{n}-q\right\|=c .
$$

Moreover, $\left\|S x_{n}-q\right\| \leq\left\|x_{n}-q\right\|$ implies that

$$
\limsup _{n \rightarrow \infty}\left\|S x_{n}-q\right\| \leq c \text {. }
$$

Thus,

$$
\begin{aligned}
c & =\lim _{n \rightarrow \infty}\left\|z_{n}-q\right\|=\lim _{n \rightarrow \infty}\left\|\gamma_{n} x_{n}+\left(1-\gamma_{n}\right) S x_{n}-q\right\| \\
& =\lim _{n \rightarrow \infty}\left\|\gamma_{n}\left(x_{n}-q\right)+\left(1-\gamma_{n}\right)\left(S x_{n}-q\right)\right\|,
\end{aligned}
$$

given by Lemma 2 that

$$
\lim _{n \rightarrow \infty}\left\|S x_{n}-x_{n}\right\|=0 .
$$

By (10) and $\sum \alpha_{n}<\infty$, then we have

$$
\begin{aligned}
\left\|x_{n+m}-q\right\| & \leq\left(1+2 \alpha_{n+m-1}\right)\left\|x_{n+m-1}-q\right\| \\
& \leq e^{2 \alpha_{n+m-1}}\left\|x_{n+m-1}-q\right\| \\
& \leq \cdots \\
& \leq e^{2 \sum_{i=n}^{n+m-1} \alpha_{i}}\left\|x_{n}-q\right\| .
\end{aligned}
$$

That is,

$$
\left\|x_{n+m}-q\right\| \leq M\left\|x_{n}-q\right\|,
$$

where $M=e^{2 \sum_{i=n}^{n+m-1} \alpha_{i}}$ for all $m, n \in \mathbb{N}$, for all $q \in F$ and for $M>0$.

Next, we prove that $\left\{x_{n}\right\}_{n=0}^{\infty}$ is a Cauchy sequence.

Since $q \in F$ arbitrarily and $\lim _{n \rightarrow \infty}\left\|x_{n}-q\right\|$ exists, consequently, $d\left(x_{n}, F\right)$ exists by Lemma 3 . From Lemma 3 and (16), we get

$$
\lim _{n \rightarrow \infty} f\left(d\left(x_{n}, F\right)\right) \leq \lim _{n \rightarrow \infty}\left\|x_{n}-S x_{n}\right\|=0 .
$$

Since $f:[0, \infty) \rightarrow[0, \infty)$ is a nondecreasing function satisfying $f(0)=0, f(r)>0$ for all $r \in(0, \infty)$, therefore, we have $\lim _{n \rightarrow \infty} d\left(x_{n}, F\right)=0$.

Let $\varepsilon>0$; since $\lim _{n \rightarrow \infty} d\left(x_{n}, F\right)=0$, therefore, there exists a constant $n_{0}$ such that, for all $n \geq n_{0}$, we have $d\left(x_{n}, F\right) \leq \varepsilon / 2 M$. There must exist $p_{1} \in F$, such that

$$
d\left(x_{n}, p_{1}\right) \leq \frac{\varepsilon}{2 M} .
$$

From (18), it can be obtained that, when $n \geq n_{0}$ and $m \in \mathbb{N}$,

$$
\begin{aligned}
\left\|x_{n+m}-x_{n}\right\| & \leq\left\|x_{n+m}-p_{1}\right\|+\left\|x_{n}-p_{1}\right\| \\
& \leq 2 M\left\|x_{n}-p_{1}\right\| \\
& \leq 2 M \cdot \frac{\varepsilon}{2 M}=\varepsilon .
\end{aligned}
$$

This implies that $\left\{x_{n}\right\}_{n=0}^{\infty}$ is a Cauchy sequence in a closed convex subset $C$ of a Banach space $E$. Thus, it must converge to a point in $C$; let $\lim _{n \rightarrow \infty} x_{n}=p$.

For all $\epsilon>0$, as $\lim _{n \rightarrow \infty} x_{n}=p$, thus, there exists a number $n_{1}$ such that, when $n_{2} \geq n_{1}$,

$$
\left\|x_{n_{2}}-p\right\| \leq \frac{\epsilon}{4} \text {. }
$$

In fact, $\lim _{n \rightarrow \infty} d\left(x_{n}, F\right)=0$ implies that using number $n_{2}$ above, when $n \geq n_{2}$, we have $d\left(x_{n}, F\right) \leq \epsilon / 8$. In particular, $d\left(x_{n_{2}}, F\right) \leq \epsilon / 8$. Thus, there must exist $\bar{p} \in F$, such that

$$
\left\|x_{n_{2}}-\bar{p}\right\|=d\left(x_{n_{2}}, \bar{p}\right) \leq \frac{\epsilon}{8} .
$$

From (22) and (23), we get

$$
\begin{aligned}
\| S & -p \| \\
& =\left\|S p-\bar{p}+S x_{n_{2}}-\bar{p}+\bar{p}-x_{n_{2}}+x_{n_{2}}-p+\bar{p}-S x_{n_{2}}\right\| \\
& \leq\|S p-\bar{p}\|+\left\|x_{n_{2}}-\bar{p}\right\|+\left\|x_{n_{2}}-p\right\|+2\left\|S x_{n_{2}}-\bar{p}\right\| \\
& \leq\|p-\bar{p}\|+3\left\|x_{n_{2}}-\bar{p}\right\|+\left\|x_{n_{2}}-p\right\| \\
& \leq 4\left\|x_{n_{2}}-\bar{p}\right\|+2\left\|x_{n_{2}}-p\right\| \\
& \leq \frac{4 \epsilon}{8}+\frac{2 \epsilon}{4}=\epsilon .
\end{aligned}
$$

As $\epsilon$ is an arbitrary positive number, thus, $S p=p$.

Let

$$
u_{n+1}=\gamma_{n} x_{n}+\beta_{n} T x_{n}+\left(1-\beta_{n}-\gamma_{n}\right) T x_{n} .
$$

Then we have

$$
\begin{aligned}
&\left\|u_{n+1}-q\right\|^{2} \\
&=\left\|\gamma_{n} x_{n}+\beta_{n} T x_{n}+\left(1-\beta_{n}-\gamma_{n}\right) T x_{n}-q\right\|^{2} \\
& \leq \gamma_{n}\left\|x_{n}-q\right\|^{2}+\beta_{n}\left\|T x_{n}-q\right\|^{2} \\
&+\left(1-\beta_{n}-\gamma_{n}\right)\left\|T x_{n}-q\right\|^{2}-\gamma_{n} \beta_{n} \varphi\left\|x_{n}-T x_{n}\right\| \\
& \leq\left\|x_{n}-q\right\|^{2}-\gamma_{n} \beta_{n} \varphi\left\|x_{n}-T x_{n}\right\| ;
\end{aligned}
$$

hence,

$$
\gamma_{n} \beta_{n} \varphi\left\|x_{n}-T x_{n}\right\| \leq\left\|x_{n}-q\right\|^{2}-\left\|u_{n+1}-q\right\|^{2},
$$

for $q \in F$. Summing from $n=1$ to $\infty$, we have

$$
\begin{aligned}
& \sum_{n=1}^{\infty} \gamma_{n} \beta_{n} \varphi\left\|x_{n}-T x_{n}\right\| \\
& \leq \sum_{n=1}^{\infty}\left(\left\|x_{n}-q\right\|^{2}-\left\|u_{n+1}-q\right\|^{2}\right) \\
& \quad \leq 2 K \sum_{n=1}^{\infty}\left\|u_{n+1}-x_{n}\right\| \\
& \quad \leq 4 K^{2} \sum_{n=1}^{\infty}\left(1-\gamma_{n}\right),
\end{aligned}
$$


where $K=\sup _{n \in \mathbb{N}}\left\{\left\|x_{n}-q\right\|\right\} ;$ since $\sum_{n=1}^{\infty}\left(1-\gamma_{n}\right)<\infty$, we get

$$
\sum_{n=1}^{\infty} \gamma_{n} \beta_{n} \varphi\left\|x_{n}-T x_{n}\right\| \leq 4 K^{2} \sum_{n=1}^{\infty}\left(1-\gamma_{n}\right)<\infty .
$$

Since $\sum_{n=1}^{\infty} \gamma_{n} \beta_{n}=\infty$, from Lemma 5, we get $\liminf _{n \rightarrow \infty} \varphi\left\|T x_{n}-x_{n}\right\|=0$. Hence,

$$
\liminf _{n \rightarrow \infty}\left\|T x_{n}-x_{n}\right\|=0
$$

Since $T$ and $R$ are nonexpansive mappings, we have

$$
\begin{aligned}
&\left\|T x_{n+1}-x_{n+1}\right\| \\
&=\| T x_{n+1}-R T x_{n}+R T x_{n}+\alpha_{n} R T x_{n} \\
& \quad-\alpha_{n} R T x_{n}-\alpha_{n} y_{n}-\left(1-\alpha_{n}\right) R z_{n} \| \\
& \leq\left\|T x_{n+1}-R T x_{n}\right\|+\left(1-\alpha_{n}\right)\left\|R T x_{n}-R z_{n}\right\| \\
& \quad+\alpha_{n}\left\|R T x_{n}-y_{n}\right\| \\
& \leq\left\|x_{n+1}-R x_{n}\right\|+\left(1-\alpha_{n}\right)\left\|T x_{n}-z_{n}\right\| \\
& \quad+\alpha_{n}\left\|R T x_{n}-y_{n}\right\| \\
& \leq \alpha_{n}\left\|y_{n}-R x_{n}\right\|+2\left(1-\alpha_{n}\right)\left\|z_{n}-x_{n}\right\| \\
&+\left(1-\alpha_{n}\right)\left\|T x_{n}-x_{n}\right\| \\
&+\alpha_{n}\left\|T x_{n}-y_{n}\right\|+\alpha_{n}\left\|R x_{n}-x_{n}\right\| \\
& \leq \alpha_{n}\left\|y_{n}-x_{n}\right\|+2 \alpha_{n}\left\|R x_{n}-x_{n}\right\| \\
&+2\left(1-\alpha_{n}\right)\left(1-\gamma_{n}\right)\left\|S x_{n}-x_{n}\right\| \\
&+\left(1-\alpha_{n}\right)\left\|T x_{n}-x_{n}\right\|+\alpha_{n} \beta_{n}\left\|T x_{n}-x_{n}\right\| \\
& \leq\left\|T x_{n}-x_{n}\right\|+2 \alpha_{n}\left\|R x_{n}-x_{n}\right\| \\
&+2\left(1-\alpha_{n}\right)\left(1-\gamma_{n}\right)\left\|S x_{n}-x_{n}\right\| .
\end{aligned}
$$

Since $\sum \alpha_{n}<\infty, \sum_{n=1}^{\infty}\left(1-\gamma_{n}\right)<\infty$, it follows from Lemma 1 that $\lim _{n \rightarrow \infty}\left\|T x_{n}-x_{n}\right\|$ exists. Therefore, from (30), we get

$$
\lim _{n \rightarrow \infty}\left\|T x_{n}-x_{n}\right\|=0
$$

Let

$$
v_{n+1}=\gamma_{n} x_{n}+\beta_{n} R x_{n}+\left(1-\beta_{n}-\gamma_{n}\right) R x_{n} .
$$

Using the same argument we can get

$$
\liminf _{n \rightarrow \infty}\left\|R x_{n}-x_{n}\right\|=0
$$

Since $R$ is a nonexpansive mapping, we have

$$
\begin{aligned}
& \left\|R x_{n+1}-x_{n+1}\right\| \\
& =\| R x_{n+1}-R x_{n}+R x_{n}+\alpha_{n} R x_{n} \\
& -\alpha_{n} R x_{n}-\alpha_{n} y_{n}-\left(1-\alpha_{n}\right) R z_{n} \| \\
& \leq\left\|x_{n+1}-x_{n}\right\|+\left(1-\alpha_{n}\right)\left\|x_{n}-z_{n}\right\| \\
& +\alpha_{n}\left\|R x_{n}-x_{n}\right\|+\alpha_{n}\left\|y_{n}-x_{n}\right\| \\
& \leq \alpha_{n}\left\|R x_{n}-x_{n}\right\|+2 \alpha_{n}\left\|y_{n}-x_{n}\right\| \\
& +\left(1-\alpha_{n}\right)\left\|R z_{n}-x_{n}\right\|+\left(1-\alpha_{n}\right)\left\|z_{n}-x_{n}\right\| \\
& \leq\left\|R x_{n}-x_{n}\right\|+2 \alpha_{n}\left\|y_{n}-x_{n}\right\|+2\left(1-\alpha_{n}\right)\left\|z_{n}-x_{n}\right\| \\
& =\left\|R x_{n}-x_{n}\right\|+2 \alpha_{n}\left(1-\beta_{n}\right)\left\|T x_{n}-x_{n}\right\| \\
& +2\left(1-\alpha_{n}\right)\left(1-\gamma_{n}\right)\left\|S x_{n}-x_{n}\right\| \text {. }
\end{aligned}
$$

Since $\sum \alpha_{n}<\infty, \sum\left(1-\gamma_{n}\right)<\infty$, it follows from Lemma 1 that $\lim _{n \rightarrow \infty}\left\|R x_{n}-x_{n}\right\|$ exists. Therefore, from (34), we get

$$
\lim _{n \rightarrow \infty}\left\|R x_{n}-x_{n}\right\|=0
$$

Then using the same argument, we can show that $\left\{x_{n}\right\}_{n=0}^{\infty}$ converges strongly to a common fixed point of $T, S$, and $R$.

\section{Convergence to a Common Fixed Point of Asymptotically Nonexpansive Mappings}

4.1. There Exists One Sequence $\left\{x_{n}\right\}$. In this part, we prove our main theorem for finding a common fixed point of asymptotically nonexpansive mappings in Banach space in the case of one sequence.

Theorem 8. Let $C$ be a nonempty closed convex subset of a uniformly convex Banach space $E$, and let $T$ and $S$ be two asymptotically nonexpansive mappings of $C$ satisfying Condition (I) and $\bar{F} \neq \emptyset$. Given $\left\{\alpha_{n}\right\},\left\{\beta_{n}\right\}$, and $\left\{\gamma_{n}\right\}$ are sequences in $(0,1)$ and $\left\{k_{n}\right\}$ with $k_{n} \geq 1$ such that $\sum \alpha_{n}<\infty$, $\sum\left(1-\beta_{n}\right)<\infty$ and $\sum\left(k_{n}-1\right)<\infty$ for all $n \geq 1$.

Define a sequence $\left\{x_{n}\right\}_{n=0}^{\infty}$ in $C$ by algorithm (3); then $\left\{x_{n}\right\}_{n=0}^{\infty}$ strongly converges to a common fixed point of $T$ and $S$.

Proof. First, we observe that $\left\{x_{n}\right\}$ is bounded; if we take an arbitrary fixed point $q$ of $\bar{F}$, noting that $\left\|y_{n}-q\right\| \leq k_{n}\left\|x_{n}-q\right\|$ and $\left\|z_{n}-q\right\| \leq k_{n}\left\|x_{n}-q\right\|$, we have

$$
\begin{aligned}
\left\|x_{n+1}-q\right\| & =\left\|\alpha_{n} y_{n}+\left(1-\alpha_{n}\right) z_{n}-q\right\| \\
& \leq \alpha_{n}\left\|y_{n}-q\right\|+\left(1-\alpha_{n}\right)\left\|Z_{n}-q\right\| \\
& \leq\left(1+k_{n}-1\right)\left\|x_{n}-q\right\| .
\end{aligned}
$$


By Lemma 1 and $\sum\left(k_{n}-1\right)<\infty$, thus, $\lim _{n \rightarrow \infty}\left\|x_{n}-q\right\|$ exists. Denote $\lim _{n \rightarrow \infty}\left\|x_{n}-q\right\|=c$, and put $k_{\infty}=\sup \left\{k_{n}: n \geq 1\right\}<$ $\infty$. Hence, $\left\{x_{n}\right\}$ is bounded, so are $\left\{y_{n}\right\}$ and $\left\{z_{n}\right\}$. Now

$$
\begin{aligned}
\left\|x_{n+1}-q\right\| & =\left\|\alpha_{n} y_{n}+\left(1-\alpha_{n}\right) z_{n}-q\right\| \\
& \leq \alpha_{n}\left\|y_{n}-z_{n}\right\|+\left\|z_{n}-q\right\| .
\end{aligned}
$$

Since $\left\|z_{n}-q\right\| \leq k_{n}\left\|x_{n}-q\right\|$, this implies that

$$
\lim _{n \rightarrow \infty}\left\|z_{n}-q\right\|=\lim _{n \rightarrow \infty}\left\|x_{n}-q\right\|=c .
$$

Moreover, $\left\|S^{n} x_{n}-q\right\| \leq k_{n}\left\|x_{n}-q\right\|$ implies that

$$
\limsup _{n \rightarrow \infty}\left\|S^{n} x_{n}-q\right\| \leq c \text {. }
$$

Thus,

$$
\begin{aligned}
c & =\lim _{n \rightarrow \infty}\left\|z_{n}-q\right\|=\lim _{n \rightarrow \infty}\left\|\gamma_{n} x_{n}+\left(1-\gamma_{n}\right) S^{n} x_{n}-q\right\| \\
& =\lim _{n \rightarrow \infty}\left\|\gamma_{n}\left(x_{n}-q\right)+\left(1-\gamma_{n}\right)\left(S^{n} x_{n}-q\right)\right\|,
\end{aligned}
$$

given by Lemma 2 that

$$
\lim _{n \rightarrow \infty}\left\|S^{n} x_{n}-x_{n}\right\|=0 .
$$

Now,

$$
\begin{aligned}
\left\|z_{n}-x_{n}\right\| & =\left\|\gamma_{n} x_{n}+\left(1-\gamma_{n}\right) S^{n} x_{n}-x_{n}\right\| \\
& \leq\left(1-\gamma_{n}\right)\left\|\left(S^{n} x_{n}-x_{n}\right)\right\| .
\end{aligned}
$$

Hence, by (42),

$$
\lim _{n \rightarrow \infty}\left\|z_{n}-x_{n}\right\|=0
$$

Also note that

$$
\begin{aligned}
\left\|x_{n+1}-x_{n}\right\| & =\left\|\alpha_{n} y_{n}+\left(1-\alpha_{n}\right) z_{n}-x_{n}\right\| \\
& \leq \alpha_{n}\left\|y_{n}-x_{n}\right\|+\left(1-\alpha_{n}\right)\left\|z_{n}-x_{n}\right\|,
\end{aligned}
$$

so that condition $\sum \alpha_{n}<\infty$ and (44) give

$$
\lim _{n \rightarrow \infty}\left\|x_{n+1}-x_{n}\right\|=0 \text {. }
$$

Next, we show $\lim _{n \rightarrow \infty}\left\|x_{n+1}-S x_{n}\right\|=0$.

We have

$$
\begin{aligned}
& \left\|x_{n+1}-S x_{n+1}\right\| \\
& \leq\left\|x_{n+1}-S^{n+1} x_{n+1}\right\|+\left\|S^{n+1} x_{n+1}-S^{n+1} x_{n}\right\| \\
& \quad+\left\|S^{n+1} x_{n}-S x_{n+1}\right\| \\
& \leq\left\|x_{n+1}-S^{n+1} x_{n+1}\right\|+k_{\infty}\left\|x_{n+1}-x_{n}\right\| \\
& \quad+k_{\infty}\left\|S^{n} x_{n}-x_{n+1}\right\| \\
& \leq\left\|x_{n+1}-S^{n+1} x_{n+1}\right\|+2 k_{\infty}\left\|x_{n+1}-x_{n}\right\| \\
& \quad+k_{\infty}\left\|S^{n} x_{n}-x_{n}\right\| .
\end{aligned}
$$

Hence, by (42) and (46), we get

$$
\lim _{n \rightarrow \infty}\left\|x_{n}-S x_{n}\right\|=0 .
$$

By (37) we assume that $k_{n}=1+r_{n}$, so $\sum r_{n}<\infty$ for $\sum\left(k_{n}-1\right)<$ $\infty$, and now,

$$
\begin{aligned}
\left\|x_{n+m}-q\right\| & \leq\left(1+r_{n+m-1}\right)\left\|x_{n+m-1}-q\right\| \\
& \leq e^{r_{n+m-1}}\left\|x_{n+m-1}-q\right\| \\
& \leq \cdots \\
& \leq e^{\sum_{i=n}^{n+m-1} r_{i}}\left\|x_{n}-q\right\| .
\end{aligned}
$$

That is,

$$
\left\|x_{n+m}-q\right\| \leq M\left\|x_{n}-q\right\|,
$$

where $M=e^{\sum_{i=n}^{n+m-1} r_{i}}$, for all $m, n \in \mathbb{N}$, for all $q \in \bar{F}$ and for $M>0$.

Next, we prove that $\left\{x_{n}\right\}_{n=0}^{\infty}$ is a Cauchy sequence.

As proved in Theorem 7 , it is easy to see that $\left\{x_{n}\right\}_{n=0}^{\infty}$ is a Cauchy sequence in a closed convex subset $C$ of a Banach space $E$. Thus, it must converge to a point in $C$; let $\lim _{n \rightarrow \infty} x_{n}=p$.

For all $\epsilon>0$, as $\lim _{n \rightarrow \infty} x_{n}=p$, thus, there exists a number $n_{1}$ such that, when $n_{2} \geq n_{1}$,

$$
\left\|x_{n_{2}}-p\right\| \leq \frac{\epsilon}{2+2 k_{\infty}}
$$

In fact, $\lim _{n \rightarrow \infty} d\left(x_{n}, \bar{F}\right)=0$ implies that using number $n_{2}$ above, when $n \geq n_{2}$, we get $d\left(x_{n}, \bar{F}\right) \leq \epsilon /\left(2+6 k_{\infty}\right)$. In particular, $d\left(x_{n_{2}}, \bar{F}\right) \leq \epsilon /\left(2+6 k_{\infty}\right)$. Thus, there must exist $\bar{p} \in \bar{F}$, such that

$$
\left\|x_{n_{2}}-\bar{p}\right\|=d\left(x_{n_{2}}, \bar{p}\right)=\frac{\epsilon}{2+6 k_{\infty}} .
$$

From (51) and (52), using the same argument in Theorem 7 , we get $S p=p$.

Now, we return to prove $\lim _{n \rightarrow \infty}\left\|x_{n}-T x_{n}\right\|=0$.

It is easy to see that $\lim _{n \rightarrow \infty}\left\|y_{n}-x_{n}\right\|=0$, and then combined with (44), we have $\lim _{n \rightarrow \infty}\left\|z_{n}-y_{n}\right\|=0$; thus, $\lim _{n \rightarrow \infty}\left\|z_{n}-q\right\|=\lim _{n \rightarrow \infty}\left\|y_{n}-q\right\|$, and then we get

$$
\lim _{n \rightarrow \infty}\left\|y_{n}-q\right\|=\lim _{n \rightarrow \infty}\left\|x_{n}-q\right\|=c .
$$

Moreover, $\left\|T^{n} x_{n}-q\right\| \leq k_{n}\left\|x_{n}-q\right\|$ implies that

$$
\limsup _{n \rightarrow \infty}\left\|T^{n} x_{n}-q\right\| \leq c ;
$$

thus,

$$
\lim _{n \rightarrow \infty}\left\|x_{n}-T^{n} x_{n}\right\|=0
$$

Therefore, we have

$$
\lim _{n \rightarrow \infty}\left\|x_{n}-T x_{n}\right\|=0 .
$$

Thus, we can show that $\left\{x_{n}\right\}_{n=0}^{\infty}$ converges strongly to a common fixed point of $T$ and $S$ immediately. 
4.2. There Exist Two Sequences $\left\{x_{n}^{\prime}\right\}$ and $\left\{x_{n}^{\prime \prime}\right\}$. In this part, we prove our main theorem for finding a common fixed point of asymptotically nonexpansive mappings in Banach space in the case of two sequences.

Theorem 9. Let $C$ be a nonempty bounded closed convex subset of a uniformly convex Banach space $E$, and let $T$ and $S$ be two asymptotically nonexpansive mappings of $C$ satisfying Condition (I) and $\bar{F} \neq \emptyset$. Given $\left\{\alpha_{n}\right\},\left\{\beta_{n}\right\}$, and $\left\{\gamma_{n}\right\}$ are sequences in $(0,1)$ and $\left\{k_{n}\right\}$ with $1 \leq k_{n}<\infty$ such that $\sum \alpha_{n}<\infty, \sum \beta_{n}<\infty, \beta_{n}>\gamma_{n}$ and $\sum\left(k_{n}-1\right)<\infty$ for all $n \geq 1$.

Define two sequences $\left\{x_{n}^{\prime}\right\}_{n=0}^{\infty}$ and $\left\{x_{n}^{\prime \prime}\right\}_{n=0}^{\infty}$ in $C$ by the algorithm (5); then $\left\{x_{n}^{\prime}\right\}_{n=0}^{\infty}$ and $\left\{x_{n}^{\prime \prime}\right\}_{n=0}^{\infty}$ strongly converge to a common fixed point of $T$ and $S$.

Proof. By the boundedness of $C$, we obverse that both $\left\{x_{n}^{\prime}\right\}$ and $\left\{x_{n}^{\prime \prime}\right\}$ are bounded; if we take an arbitrary fixed point $q$ of $\bar{F}$, noting that $\left\|y_{n}-q\right\| \leq k_{n}\left\|x_{n}^{\prime}-q\right\|$ and $\left\|z_{n}-q\right\| \leq k_{n}\left\|x_{n}^{\prime \prime}-q\right\|$, we have

$$
\begin{aligned}
\left\|x_{n+1}^{\prime}-q\right\|= & \left\|\alpha_{n} z_{n}+\left(1-\alpha_{n}\right) y_{n}-q\right\| \\
\leq & \alpha_{n}\left\|z_{n}-y_{n}\right\|+\left\|y_{n}-q\right\| \\
= & \left(1+\alpha_{n} k_{n}+k_{n}-1\right)\left\|x_{n}^{\prime}-q\right\| \\
& +\alpha_{n} k_{n}\left\|x_{n}^{\prime \prime}-q\right\| .
\end{aligned}
$$

By Lemma 1 and $\sum \alpha_{n}<\infty, \sum\left(k_{n}-1\right)<\infty$, thus, $\lim _{n \rightarrow \infty}\left\|x_{n}^{\prime}-q\right\|$ exists. Denote

$$
\lim _{n \rightarrow \infty}\left\|x_{n}^{\prime}-q\right\|=c^{\prime} .
$$

Similarly, we have

$$
\lim _{n \rightarrow \infty}\left\|x_{n}^{\prime \prime}-q\right\|=c^{\prime \prime} .
$$

Since both $\left\{x_{n}^{\prime}\right\}$ and $\left\{x_{n}^{\prime \prime}\right\}$ are bounded, put $k_{\infty}=\sup \left\{k_{n}\right.$ : $n \geq 1\}<\infty$. We get that $\left\{y_{n}\right\}$ and $\left\{z_{n}\right\}$ are bounded. Now

$$
\begin{aligned}
\left\|x_{n+1}^{\prime}-q\right\| & =\left\|\alpha_{n} z_{n}+\left(1-\alpha_{n}\right) y_{n}-q\right\| \\
& \leq \alpha_{n}\left\|z_{n}-y_{n}\right\|+\left\|y_{n}-q\right\| .
\end{aligned}
$$

Since $\left\|y_{n}-q\right\| \leq k_{n}\left\|x_{n}^{\prime}-q\right\|$, this implies that

$$
\lim _{n \rightarrow \infty}\left\|y_{n}-q\right\|=\lim _{n \rightarrow \infty}\left\|x_{n}^{\prime}-q\right\|=c^{\prime} .
$$

Moreover, $\left\|T^{n} x_{n}^{\prime}-q\right\| \leq k_{n}\left\|x_{n}^{\prime}-q\right\|$ implies that

$$
\limsup _{n \rightarrow \infty}\left\|T^{n} x_{n}^{\prime}-q\right\| \leq c^{\prime} .
$$

Thus,

$$
\begin{aligned}
c^{\prime} & =\lim _{n \rightarrow \infty}\left\|y_{n}-q\right\| \\
& =\lim _{n \rightarrow \infty}\left\|\beta_{n} x_{n}^{\prime}+\left(1-\beta_{n}\right) T^{n} x_{n}^{\prime}-q\right\| \\
& =\lim _{n \rightarrow \infty}\left\|\beta_{n}\left(x_{n}^{\prime}-q\right)+\left(1-\beta_{n}\right)\left(T^{n} x_{n}^{\prime}-q\right)\right\|,
\end{aligned}
$$

given by Lemma 2 that

$$
\lim _{n \rightarrow \infty}\left\|T^{n} x_{n}^{\prime}-x_{n}^{\prime}\right\|=0 .
$$

Also note that

$$
\begin{aligned}
& \left\|x_{n+1}^{\prime}-x_{n}^{\prime}\right\| \\
& \quad=\left\|\alpha_{n} z_{n}+\left(1-\alpha_{n}\right) y_{n}-x_{n}^{\prime}\right\| \\
& \quad \leq \alpha_{n}\left\|z_{n}-y_{n}\right\|+\left(1-\beta_{n}\right)\left\|\left(T^{n} x_{n}^{\prime}-x_{n}^{\prime}\right)\right\|,
\end{aligned}
$$

so that condition $\sum \alpha_{n}<\infty$ and (64) give

$$
\lim _{n \rightarrow \infty}\left\|x_{n+1}^{\prime}-x_{n}^{\prime}\right\|=0 \text {. }
$$

We have

$$
\begin{aligned}
& \left\|x_{n+1}^{\prime}-T x_{n+1}^{\prime}\right\| \\
& \leq\left\|x_{n+1}^{\prime}-T^{n+1} x_{n+1}^{\prime}\right\|+\left\|T^{n+1} x_{n+1}^{\prime}-T^{n+1} x_{n}^{\prime}\right\| \\
& \quad+\left\|T^{n+1} x_{n}^{\prime}-T x_{n+1}^{\prime}\right\| \\
& \leq\left\|x_{n+1}^{\prime}-T^{n+1} x_{n+1}^{\prime}\right\| \\
& \quad+k_{\infty}\left\|x_{n+1}^{\prime}-x_{n}^{\prime}\right\|+k_{\infty}\left\|T^{n} x_{n}^{\prime}-x_{n+1}^{\prime}\right\| \\
& \leq\left\|x_{n+1}^{\prime}-T^{n+1} x_{n+1}^{\prime}\right\|+2 k_{\infty}\left\|x_{n+1}^{\prime}-x_{n}^{\prime}\right\| \\
& \quad+k_{\infty}\left\|T^{n} x_{n}^{\prime}-x_{n}^{\prime}\right\| .
\end{aligned}
$$

Hence, by (64) and (66), we get

$$
\lim _{n \rightarrow \infty}\left\|x_{n}^{\prime}-T x_{n}^{\prime}\right\|=0,
$$

and then we assume that $k_{n}=1+r_{n}$, so $\sum r_{n}<\infty$ for $\sum\left(k_{n}-\right.$ $1)<\infty$; now by (57), we obtain that

$$
\begin{aligned}
& \left\|x_{n+m}^{\prime}-q\right\| \\
& \leq\left(1+\alpha_{n+m-1}\right)\left(1+r_{n+m-1}\right)\left\|x_{n+m-1}^{\prime}-q\right\|+s_{n+m-1} \\
& \leq e^{\alpha_{n+m-1}} e^{r_{n+m-1}}\left\|x_{n+m-1}^{\prime}-q\right\|+s_{n+m-1} \\
& \leq \ldots \\
& \leq e^{\sum_{i=n}^{n+m-1} \alpha_{i}} e^{\sum_{i=n}^{n+m-1} r_{i}}\left\|x_{n}^{\prime}-q\right\| \\
& \quad+e^{\sum_{i=n}^{n+m-1} \alpha_{i}} e^{\sum_{i=n}^{n+m-1} r_{i}} \sum_{i=n}^{n+m-1} s_{i},
\end{aligned}
$$

where $s_{i}=\alpha_{i} k_{i}\left\|x_{n}^{\prime \prime}-q\right\|$.

By $\sum \alpha_{n}<\infty$ and the convergence of $\left\{r_{n}\right\}$, that is,

$$
\left\|x_{n+m}^{\prime}-q\right\| \leq M\left(\left\|x_{n}^{\prime}-q\right\|+\sum_{i=n}^{\infty} s_{i}\right),
$$

where $M=e^{\sum_{i=n}^{n+m-1} \alpha_{i}} e^{\sum_{i=n}^{n+m-1} r_{i}}, s_{i}=\alpha_{i} k_{i}\left\|x_{n}^{\prime \prime}-q\right\|$, for all $m, n \in$ $\mathbb{N}, q \in \bar{F}$ and for $M>0$.

Next, we prove that $\left\{x_{n}^{\prime}\right\}_{n=0}^{\infty}$ is a Cauchy sequence. 
Since $q \in \bar{F}$ arbitrarily and $\lim _{n \rightarrow \infty}\left\|x_{n}^{\prime}-q\right\|$ exists, consequently, $d\left(x_{n}^{\prime}, \bar{F}\right)$ exists by Lemma 3 . From Lemma 3 and (68), we get

$$
\lim _{n \rightarrow \infty} f\left(d\left(x_{n}^{\prime}, \bar{F}\right)\right) \leq \lim _{n \rightarrow \infty}\left\|x_{n}^{\prime}-T x_{n}^{\prime}\right\|=0 .
$$

Since $f:[0, \infty) \rightarrow[0, \infty)$ is a nondecreasing function satisfying $f(0)=0, f(r)>0$ for all $r \in(0, \infty)$, therefore, we have

$$
\lim _{n \rightarrow \infty} d\left(x_{n}^{\prime}, \bar{F}\right)=0 .
$$

Let $\varepsilon>0$, since $\lim _{n \rightarrow \infty} d\left(x_{n}^{\prime}, \bar{F}\right)=0$ and $\sum_{i=1}^{\infty} s_{i}<\infty$, therefore, there exists a constant $n_{0}$ such that, for all $n \geq n_{0}$, we have

$$
d\left(x_{n}^{\prime}, \bar{F}\right) \leq \frac{\varepsilon}{3 M}, \quad \sum_{j=n_{0}}^{\infty} s_{j} \leq \frac{\varepsilon}{6 M}
$$

in particular,

$$
d\left(x_{n_{0}}^{\prime}, \bar{F}\right) \leq \frac{\varepsilon}{3 M} .
$$

There must exist $p_{1} \in \bar{F}$ such that

$$
d\left(x_{n_{0}}^{\prime}, p_{1}\right) \leq \frac{\varepsilon}{3 M} .
$$

From (70), it can be obtained that, when $n \geq n_{0}$,

$$
\begin{aligned}
\left\|x_{n+m}^{\prime}-x_{n}^{\prime}\right\| & \leq\left\|x_{n+m}^{\prime}-p_{1}\right\|+\left\|x_{n}^{\prime}-p_{1}\right\| \\
& \leq 2 M\left(\left\|x_{n_{0}}^{\prime}-p_{1}\right\|+\sum_{j=n_{0}}^{n_{0}+m-1} s_{j}\right) \\
& \leq 2 M\left(\frac{\varepsilon}{3 M}+\frac{\varepsilon}{6 M}\right)=\varepsilon .
\end{aligned}
$$

This implies that $\left\{x_{n}^{\prime}\right\}_{n=0}^{\infty}$ is a Cauchy sequence in a closed convex subset $C$ of a Banach space $E$. Thus, it must converge to a point in $C$; let $\lim _{n \rightarrow \infty} x_{n}^{\prime}=x^{\prime}$.

For all $\epsilon>0$, on lines similar to Theorem 8 , from $\lim _{n \rightarrow \infty} x_{n}^{\prime}=x^{\prime}$, it can be proved that $\left\|T x^{\prime}-x^{\prime}\right\| \leq \epsilon$. As $\epsilon$ is an arbitrary positive number, thus, $T x^{\prime}=x^{\prime}$. Similarly, we have $\lim _{n \rightarrow \infty}\left\|S x_{n}^{\prime \prime}-x_{n}^{\prime \prime}\right\|=0$, and then $S x^{\prime \prime}=x^{\prime \prime}\left(x_{n}^{\prime \prime} \rightarrow x^{\prime \prime}\right.$ as $n \rightarrow \infty)$.

Finally, we prove $x^{\prime}=x^{\prime \prime}$.

Let $w_{n+1}=\alpha_{n} T^{n} x_{n}^{\prime}+\left(1-\alpha_{n}\right) S^{n} x_{n}^{\prime}$, and put $\left\|x_{n}^{\prime \prime}-q\right\| \geq$ $\left\|x_{n}^{\prime}-q\right\|$, for all $n \geq 1$. Then,

$$
\begin{aligned}
\left\|w_{n+1}-q\right\| \leq & \alpha_{n} k_{n}\left\|x_{n}^{\prime}-q\right\| \\
& +\left(1-\alpha_{n}\right) k_{n}\left\|x_{n}^{\prime \prime}-q\right\| \\
\leq & k_{n} \max \left\{\left\|x_{n}^{\prime}-q\right\|,\left\|x_{n}^{\prime \prime}-q\right\|\right\} \\
\leq & k_{n}\left\|x_{n}^{\prime \prime}-q\right\| .
\end{aligned}
$$

We have $\lim _{n \rightarrow \infty}\left\|w_{n+1}-q\right\| \leq \lim _{n \rightarrow \infty}\left\|x_{n}^{\prime \prime}-q\right\|$. Now,

$$
\begin{aligned}
\left\|x_{n+1}^{\prime \prime}-q\right\|= & \left\|\alpha_{n} y_{n}+\left(1-\alpha_{n}\right) z_{n}-q\right\| \\
\leq & \beta_{n}\left\|\alpha_{n} x_{n}^{\prime}+\left(1-\alpha_{n}\right) x_{n}^{\prime \prime}\right\| \\
& +\left\|\alpha_{n} T^{n} x_{n}^{\prime}+\left(1-\alpha_{n}\right) S^{n} x_{n}^{\prime \prime}-q\right\| \\
= & \beta_{n}\left\|\alpha_{n} x_{n}^{\prime}+\left(1-\alpha_{n}\right) x_{n}^{\prime \prime}\right\|+\left\|w_{n+1}-q\right\| .
\end{aligned}
$$

Since $\sum \beta_{n}<\infty$ and the boundedness of $\left\{x_{n}^{\prime}\right\}$ and $\left\{x_{n}^{\prime \prime}\right\}$, we get

$$
\lim _{n \rightarrow \infty}\left\|w_{n+1}-q\right\|=c^{\prime \prime}
$$

Moreover, $\left\|T^{n} x_{n}^{\prime}-q\right\| \leq k_{n}\left\|x_{n}^{\prime}-q\right\|$ and $\left\|S^{n} x_{n}^{\prime \prime}-q\right\| \leq k_{n}\left\|x_{n}^{\prime \prime}-q\right\|$ imply that

$$
\limsup _{n \rightarrow \infty}\left\|T^{n} x_{n}^{\prime}-q\right\| \leq c^{\prime \prime}, \quad \limsup _{n \rightarrow \infty}\left\|S^{n} x_{n}^{\prime \prime}-q\right\| \leq c^{\prime \prime} .
$$

Thus,

$$
\begin{aligned}
c^{\prime \prime} & =\lim _{n \rightarrow \infty}\left\|w_{n+1}-q\right\| \\
& =\left\|\alpha_{n} T^{n} x_{n}^{\prime}+\left(1-\alpha_{n}\right) S^{n} x_{n}^{\prime \prime}-q\right\| \\
& =\left\|\alpha_{n}\left(T^{n} x_{n}^{\prime}-q\right)+\left(1-\alpha_{n}\right)\left(S^{n} x_{n}^{\prime \prime}-q\right)\right\|,
\end{aligned}
$$

given by Lemma 2 that

$$
\lim _{n \rightarrow \infty}\left\|S^{n} x_{n}^{\prime \prime}-T^{n} x_{n}^{\prime}\right\|=0
$$

So

$$
\begin{aligned}
& \lim _{n \rightarrow \infty}\left\|x_{n+1}^{\prime \prime}-x_{n+1}^{\prime \prime}\right\| \\
& =\lim _{n \rightarrow \infty}\left|2 \alpha_{n}-1\right|\left\|z_{n}-y_{n}\right\| \\
& =\lim _{n \rightarrow \infty}\left|2 \alpha_{n}-1\right| \| \gamma_{n}\left(x_{n}^{\prime \prime}-S^{n} x_{n}^{\prime \prime}\right) \\
& \quad-\beta_{n}\left(x_{n}^{\prime}-T^{n} x_{n}^{\prime}\right)+\left(S^{n} x_{n}^{\prime \prime}-T^{n} x_{n}^{\prime}\right) \|,
\end{aligned}
$$

so we obtain that $\lim _{n \rightarrow \infty}\left\|x_{n+1}^{\prime}-x_{n+1}^{\prime \prime}\right\|=0$ for (64) and (82), and it means $x^{\prime}=x^{\prime \prime}$. This completes the proof.

\section{Application: Convergence to a Zero of Accretive Operators}

Let $E$ be a real Banach space. Recall that an operator (possibly multivalued) $A$ with domain $D(A)$ and range $R(A)$ in $E$ is said to be accretive if, for each $x_{i} \in D(A)$ and $y_{i} \in A x_{i}(i=1,2)$, there exists a $j\left(x_{1}-x_{2}\right) \in J\left(x_{1}-x_{2}\right)$ such that

$$
\left\langle y_{1}-y_{2}, j\left(x_{1}-x_{2}\right)\right\rangle \geq 0,
$$


where $J$ is the normalized duality map from $E$ to the dual space $E^{*}$ given by

$$
J(x)=\left\{x^{*} \in E^{*}:\left\langle x, x^{*}\right\rangle=\|x\|^{2}=\left\|x^{*}\right\|^{2}\right\}, \quad x \in E .
$$

An accretive operator $A$ is $m$-accretive if $R(I+r A)=E$ for all $r>0$. Denote the zero set of $A$ by

$$
F:=A^{-1}(0)=\{z \in D(A): 0 \in A z\} .
$$

For an $m$-accretive operator $A$ with $F \neq \emptyset$ and $C=\overline{D(A)}$ convex, the problem of finding a zero of $A$, that is,

$$
\text { find } z \in C \text { such that } 0 \in A z \text {, }
$$

has extensively been investigated due to its applications in related problems such as minimization problems, variational inequality problems, and nonlinear evolution equations.

It is known that the resolvent of $A$, defined by

$$
J_{r}=(I+r A)^{-1},
$$

for $r>0$, is a nonexpansive mapping from $E$ to $C$ and it is straightforward to see that $F$ coincides with the fixed point set of $J_{r}$ for any $r>0$. Therefore, (87) is equivalent to the fixed point problem $z=J_{r} z$. Then an interesting approach to solving this problem is via iterative methods for nonexpansive mappings. We need the resolvent identity [15].

Theorem 10. Let $E$ be a uniformly convex Banach space, and let $A$ be an m-accretive operator in $E$ such that $\operatorname{Fix}\left(J_{r_{1}}\right) \bigcap \operatorname{Fix}\left(J_{r_{2}}\right) \bigcap \operatorname{Fix}\left(J_{r_{3}}\right)=A^{-1}(0) \neq \emptyset, J_{r_{i}}: E \rightarrow E$ is nonexpansive commuting mappings for all $r_{i}>0(i=$ $1,2,3)$ satisfying Condition (I). Given $\left\{\alpha_{n}\right\},\left\{\beta_{n}\right\}$, and $\left\{\gamma_{n}\right\}$ are sequences in $(0,1)$, such that $\sum \alpha_{n}<\infty, \sum \gamma_{n} \beta_{n}=\infty$, $\sum\left(1-\gamma_{n}\right)<\infty$ and $r_{i, n} \geq \varepsilon$ for some $\varepsilon>0$ for all $n \geq 1$.

Define a sequence $\left\{x_{n}\right\}_{n=0}^{\infty}$ by algorithm (4); then $\left\{x_{n}\right\}_{n=0}^{\infty}$ strongly converges to a zero of $A$.

Proof. Take any arbitrary $q \in A^{-1}(0)$; it follows from Lemma 1 that $\lim _{n \rightarrow \infty}\left\|x_{n}-q\right\|$ exists. From Lemma 2 , it can be shown that $\lim _{n \rightarrow \infty}\left\|J_{r_{i, n}} x_{n}-x_{n}\right\|=0(i=1,2,3)$. Since $J_{r_{i}}: E \rightarrow$ $E$ is nonexpansive for all $r_{i}>0$, it follows from Lemma 6 that $\lim _{n \rightarrow \infty}\left\|J_{r_{i}} x_{n}-x_{n}\right\|=0$. Therefore, all the conditions in Theorem 7 are satisfied. The conclusion of Theorem 10 can be obtained from Theorem 7 immediately.

Theorem 11. Let $C$ be a nonempty bounded closed convex subset of a uniformly convex Banach space $E$, and let $A$ be an m-accretive operator in $C$ such that $\operatorname{Fix}\left(J_{r_{1}}\right) \bigcap \operatorname{Fix}\left(J_{r_{2}}\right)=$ $A^{-1}(0) \neq \emptyset, J_{r_{i}}: C \rightarrow C$ is nonexpansive for all $r_{i}>0$ $(i=1,2)$ satisfying Condition (I). Given $\left\{\alpha_{n}\right\},\left\{\beta_{n}\right\}$, and $\left\{\gamma_{n}\right\}$ are sequences in $(0,1)$ such that $\sum \alpha_{n}<\infty, \sum \beta_{n}<\infty, \beta_{n}>\gamma_{n}$ and $r_{i, n} \geq \varepsilon$ for some $\varepsilon>0$ for all $n \geq 1$.

Define two sequences $\left\{x_{n}^{\prime}\right\}_{n=0}^{\infty}$ and $\left\{x_{n}^{\prime \prime}\right\}_{n=0}^{\infty}$ in $C$ by algorithm (6); then $\left\{x_{n}^{\prime}\right\}_{n=0}^{\infty}$ and $\left\{x_{n}^{\prime \prime}\right\}_{n=0}^{\infty}$ strongly converge to a zero of $A$.

Proof. Only a sketch of the proof is given here.
Take any arbitrary $q \in \operatorname{Fix}\left(J_{r_{1}}\right) \bigcap \operatorname{Fix}\left(J_{r_{2}}\right)$; it follows from Lemma 1 that $\lim _{n \rightarrow \infty}\left\|x_{n}^{\prime}-q\right\|$ and $\lim _{n \rightarrow \infty}\left\|x_{n}^{\prime \prime}-q\right\|$ exist. From Lemma 2, it can be shown that $\lim _{n \rightarrow \infty}\left\|J_{r_{1, n}} x_{n}^{\prime}-x_{n}^{\prime}\right\|=0$ and $\lim _{n \rightarrow \infty}\left\|J_{r_{2, n}} x_{n}^{\prime \prime}-x_{n}^{\prime \prime}\right\|=0$. Since $J_{r_{i}}: E \rightarrow E$ is nonexpansive for all $r_{i}>0(i=1,2)$, it follows from lemma 2.6 that $\lim _{n \rightarrow \infty}\left\|J_{r_{1}} x_{n}^{\prime}-x_{n}^{\prime}\right\|=0$ and $\lim _{n \rightarrow \infty}\left\|J_{r_{2}} x_{n}^{\prime \prime}-x_{n}^{\prime \prime}\right\|=0$. Therefore, all the conditions in Theorems 7, 8, and 9, are satisfied and using the same argument in those theorems, the conclusion of Theorem 11 can be obtained immediately.

\section{Authors' Contribution}

All authors contributed equally and significantly in writing this paper. All authors read and approved the final manuscript.

\section{Conflict of Interests}

The authors declare that there is no conflict of interests regarding the publication of this paper.

\section{Acknowledgments}

This work was supported by the National Natural Science Foundation of China (Grant no. 11171046) and supported by the Scientific Research Foundation of CUIT (no. KYTZ201004).

\section{References}

[1] F. E. Browder, "Fixed-point theorems for noncompact mappings in Hilbert space," Proceedings of the National Academy of Sciences of the United States of America, vol. 53, pp. 1272-1276, 1965.

[2] K. Goebel and W. A. Kirk, "A fixed point theorem for asymptotically nonexpansive mappings," Proceedings of the American Mathematical Society, vol. 35, pp. 171-174, 1972.

[3] W. R. Mann, "Mean value methods in iteration," Proceedings of the American Mathematical Society, vol. 4, pp. 506-510, 1953.

[4] F. E. Browder and W. V. Petryshyn, "Construction of fixed points of nonlinear mappings in Hilbert space," Journal of Mathematical Analysis and Applications, vol. 20, pp. 197-228, 1967.

[5] C. Byrne, "A unified treatment of some iterative algorithms in signal processing and image reconstruction," Inverse Problems, vol. 20, no. 1, pp. 103-120, 2004.

[6] B. Halpern, "Fixed points of nonexpanding maps," Bulletin of the American Mathematical Society, vol. 73, pp. 957-961, 1967.

[7] T.-H. Kim and H.-K. Xu, "Strong convergence of modified Mann iterations," Nonlinear Analysis: Theory, Methods \& Applications, vol. 61, no. 1-2, pp. 51-60, 2005.

[8] K. Nammanee, M. A. Noor, and S. Suantai, "Convergence criteria of modified Noor iterations with errors for asymptotically nonexpansive mappings," Journal of Mathematical Analysis and Applications, vol. 314, no. 1, pp. 320-334, 2006.

[9] S. Reich, "Weak convergence theorems for nonexpansive mappings in Banach spaces," Journal of Mathematical Analysis and Applications, vol. 67, no. 2, pp. 274-276, 1979. 
[10] H. F. Senter and W. G. Dotson, Jr., "Approximating fixed points of nonexpansive mappings," Proceedings of the American Mathematical Society, vol. 44, pp. 375-380, 1974.

[11] H.-K. Xu, "Iterative algorithms for nonlinear operators," Journal of the London Mathematical Society, vol. 66, no. 1, pp. 240-256, 2002.

[12] J. Schu, "Weak and strong convergence to fixed points of asymptotically nonexpansive mappings," Bulletin of the Australian Mathematical Society, vol. 43, pp. 153-159, 1991.

[13] Y. J. Cho, H. Y. Zhou, and G. T. Guo, "Weak and strong convergence theorems for three-step iterations with errors for asymptotically nonexpansive mappings," Computers \& Mathematics with Applications, vol. 47, no. 4-5, pp. 707-717, 2004.

[14] K. S. Kim, "Approximating common fixed points of nonspreading-type mappings and nonexpansive mappings in a Hilbert space," Abstract and Applied Analysis, vol. 2012, Article ID 594218, 18 pages, 2012.

[15] V. Barbu, Nonlinear Semi-Groups and Differential Equations in Banach Space, Noordhoff, Leiden, The Netherlands, 1976. 


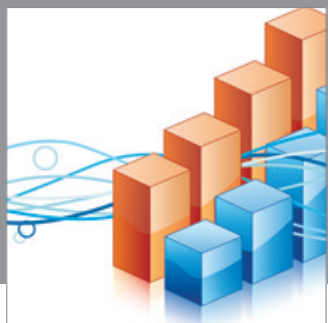

Advances in

Operations Research

mansans

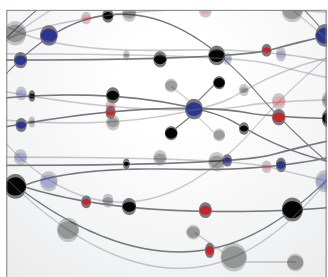

The Scientific World Journal
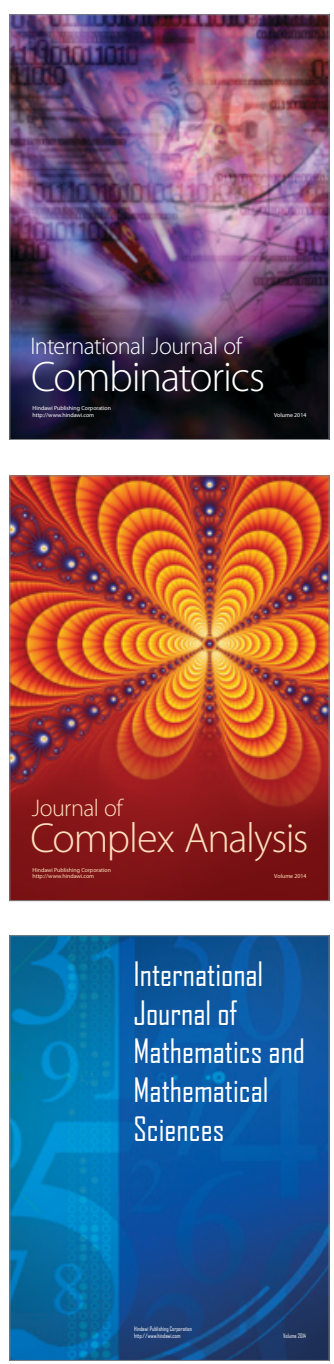
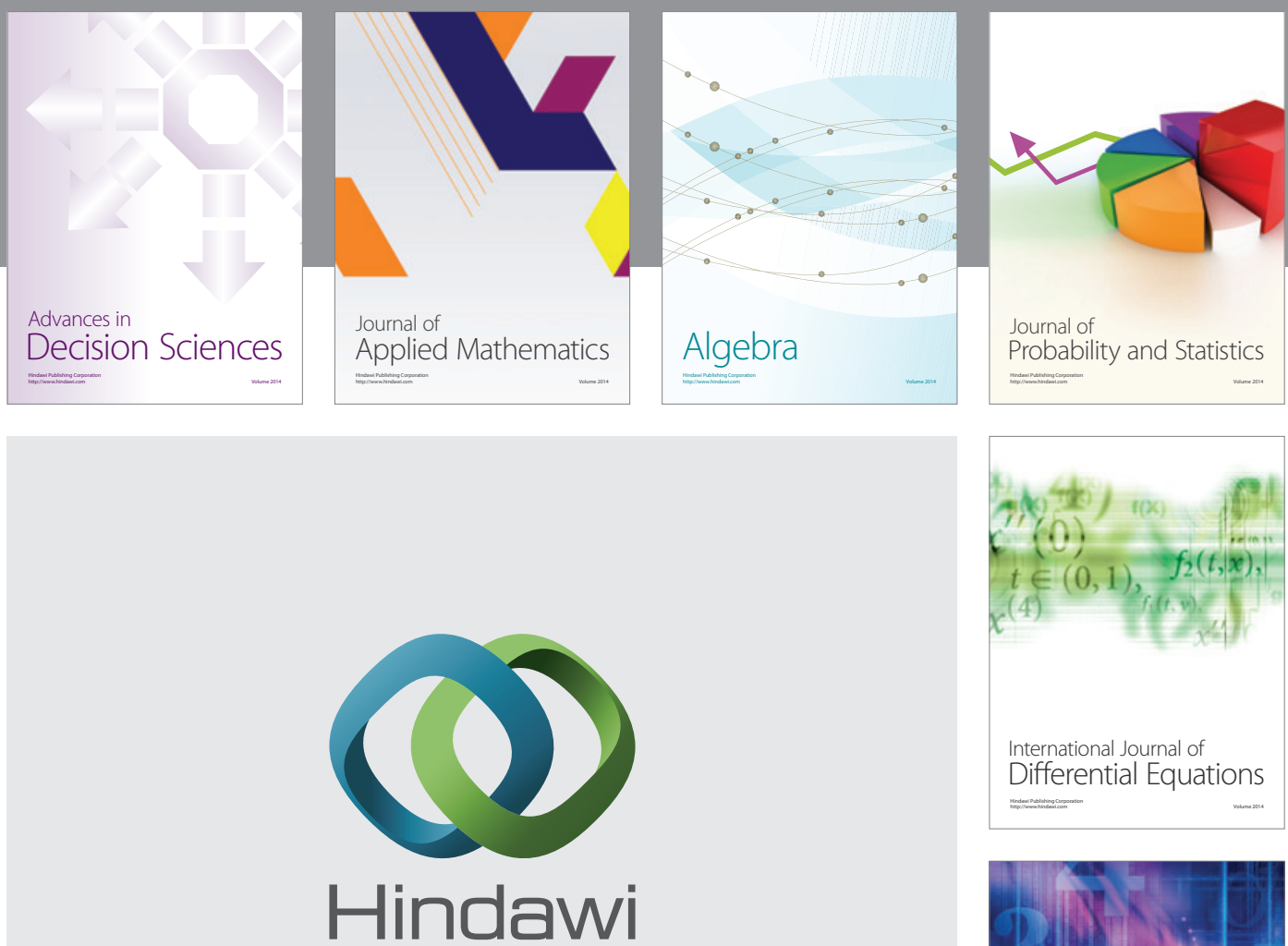

Submit your manuscripts at http://www.hindawi.com
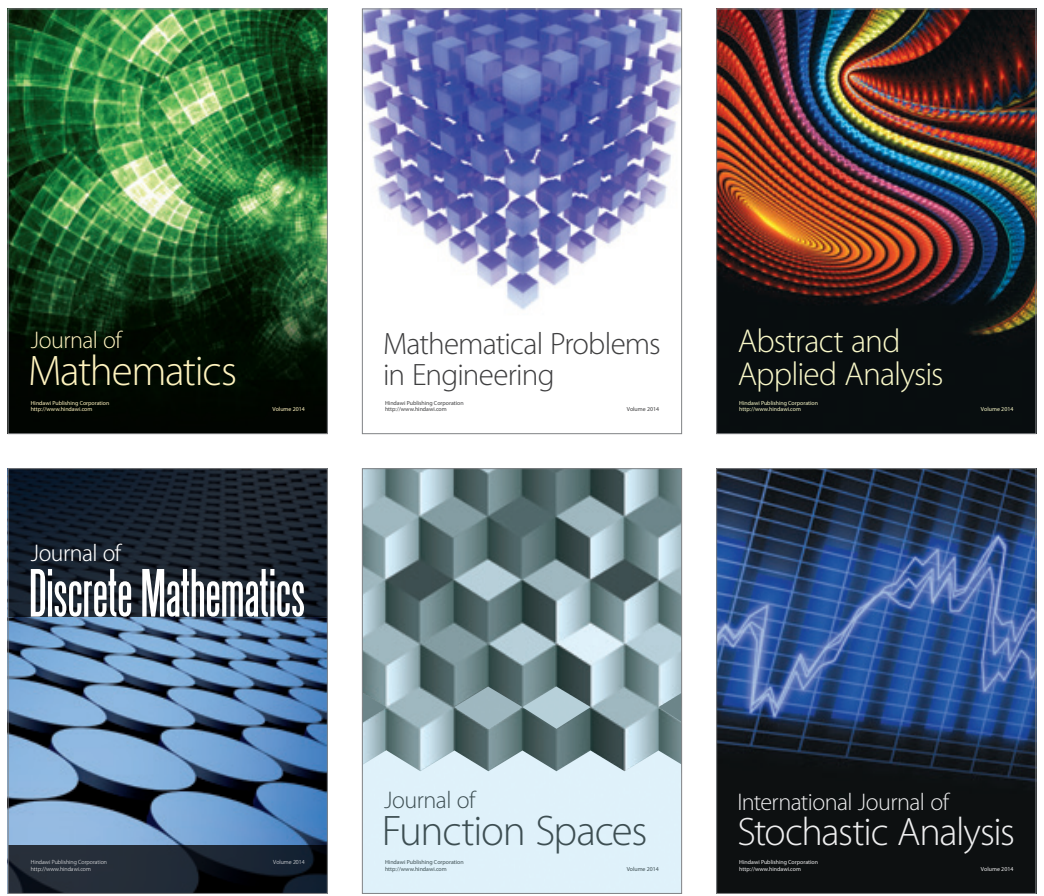

Journal of

Function Spaces

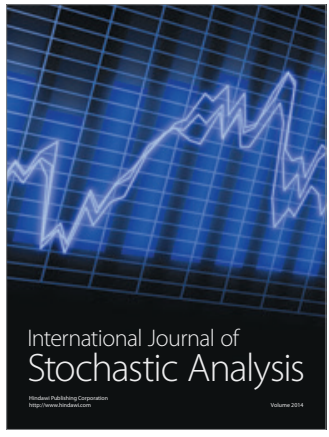

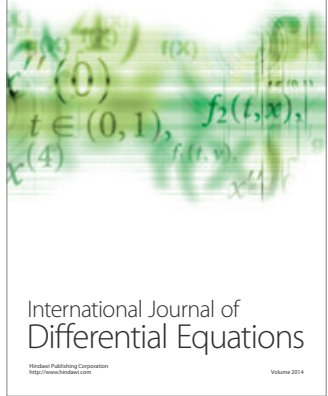
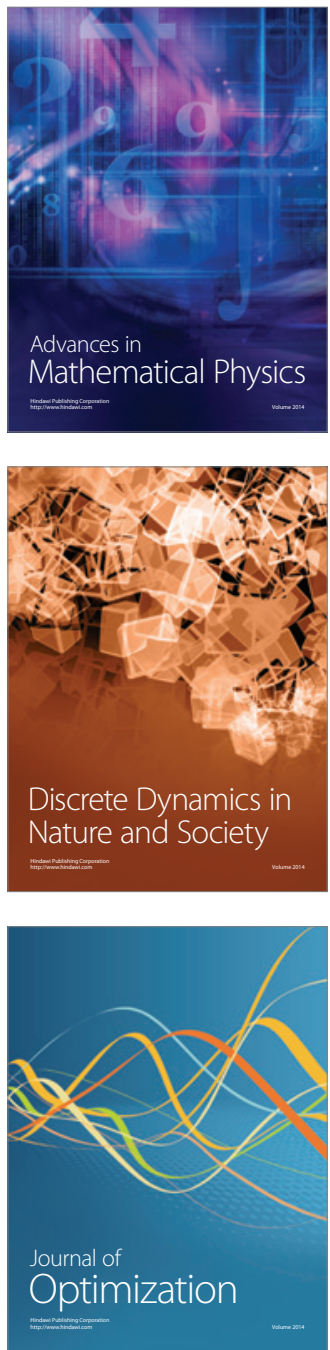